\title{
Condiciones de empleo de un grupo de trabajadores con discapacidad en Cali, Colombia*
}

\author{
Employment Conditions of a Group of Workers \\ with Disabilities in Cali, Colombia \\ Condições de emprego de um grupo de trabalhadores \\ com deficiência em Cali, Colômbia
}

\author{
MELANIA SATIZABAL REYES **
}

Universidad del Valle (Cali, Colombia)

\footnotetext{
* Este artículo es producto de la tesis de maestría en sociología de la Universidad del Valle denominada «Inserción laboral de un grupo de trabajadores con discapacidad en Cali». La investigación duró dos años y se realizó con financiación propia. Artículo de investigación recibido el 20.02.2018 y aceptado el 07.02.2019.

** Profesora de la Escuela de Rehabilitación Humana, Universidad del Valle. Email: melania.satizabal@correounivalle. edu.co ORCID: https://orcid.org/oooo-0oo3-0793-6031
} 


\section{Cómo citar}

Satizabal, Melania (2019). Condiciones de empleo de un grupo de trabajadores con discapacidad en Cali, Colombia. Revista CS, 27, 61-88. https://doi.org/10.18046/recs.i27.2773 
Resumen

Abstract

Resumo

Las personas con discapacidad han vivido situaciones de exclusión social y discriminación. Sin embargo, algunas han logrado insertarse en el mercado de trabajo. Indagar por las condiciones de empleo de un grupo de trabajadores con discapacidad es el objetivo de este artículo, para lo cual se realizó un estudio cuantitativo, descriptivo, transversal a 160 trabajadores con discapacidad en Cali. Se construyó un índice de condiciones de empleo y se encontró que uno de cada dos trabajadores presenta buenas condiciones de empleo. El tipo de discapacidad influye en la inserción laboral. La heterogeneidad del grupo revela que los trabajadores con discapacidad física presentan mejores condiciones de empleo, mientras que los trabajadores con discapacidad mental tienen las peores condiciones. La dependencia laboral influye en las condiciones de empleo, y los trabajadores asalariados presentan mejores condiciones de empleo que los independientes.

\section{PALABRAS CLAVE:}

discapacidad, empleo, mercado de trabajo

People with disabilities have experienced situations of social exclusion and discrimination. However, some have managed to join the labor market. This paper aims to explore the employment conditions of a group of workers with disabilities. A quantitative, descriptive, cross-sectional study was carried out with a sample of 160 workers with disabilities in Cali. An index of employment conditions was designed. The study found that one in two workers has good employment conditions. The type of disability impacts employability. The stark diversity of the sample reveals that physically disabled workers have better employment conditions, while mentally disabled workers have worse conditions. Labor dependence influences employment conditions. Salaried workers have better employment conditions than freelance workers.

\section{KEYWORDS:}

Disability, Employment, Labor Market 
Pessoas com deficiência sofreram situações de exclusão social e discriminação. No entanto, alguns logrado conseguiram inserir-se no mercado de trabalho. Indagar sobre as condições de emprego de um grupo de trabalhadores com deficiências é o objetivo deste artigo. Realizou-se um estudo quantitativo, descritivo e transversal em 160 trabalhadores com deficiência em Cali, na Colômbia. Construiu-se um índice de condições de emprego. Verificou-se que um em cada dois trabalhadores tem boas condições de emprego. O tipo de deficiência influencia inserção laboral. A heterogeneidade do grupo revela que os trabalhadores com deficiência física têm melhores condições de emprego, enquanto, os trabalhadores com deficiência mental têm piores condições. A dependência do trabalho influencia as condições de emprego. Os trabalhadores assalariados têm melhores condições de emprego do que os trabalhadores independentes.

\section{PALAVRAS-CHAVE:}

deficiência, emprego, mercado de trabalho 


\section{Introducción}

El grupo de personas con discapacidad incluye a aquellas que tengan deficiencias físicas, mentales, intelectuales o sensoriales a largo plazo que, al interactuar con diversas barreras, puedan impedir su participación plena y efectiva en la sociedad, en igualdad de condiciones con las demás (Naciones Unidas, 2007). Históricamente, este grupo poblacional se ha enfrentado a situaciones de exclusión y discriminación, viviendo desventajas educativas y de inserción al empleo (López y Seco, 2005; Solsona, 2018). Además, la participación en el mercado de trabajo es menor en comparación a la población sin discapacidad (Canals, 2003; Díaz, 2014; Hernández y Millán, 2015; Maldonado, 2005).

Por su parte, la mayoría de las investigaciones sobre la participación de las personas con discapacidad en el mercado de trabajo se han centrado en indagar cuáles son los canales de acceso, las estrategias para la inserción y las barreras a las que se enfrentan las personas con discapacidad, poniendo el énfasis en la población desempleada (Albarrán-Lozano y Alonso-González, 2010; Anatol'evna y Natigovna, 2017; Boiarov, 2009; Jang, Wang y Lin, 2014; Rodríguez, 2010; Rodríguez y Rico, 2009; Vornholt et al., 2018). En menor proporción, se ha investigado sobre el trabajador con discapacidad y sus condiciones de empleo. De manera que las pocas investigaciones realizadas sobre el tema presentan escasa integración de las diferentes variables que configuran las condiciones de empleo y la ausencia de todos los tipos de discapacidad. En Colombia no se encontraron investigaciones específicas sobre las condiciones de empleo de las personas con discapacidad.

Lo anterior pone en evidencia la necesidad de ahondar en términos investigativos sobre las condiciones de empleo de los trabajadores con discapacidad. Por tal razón, el presente artículo tiene como objetivo describir las condiciones de empleo de un grupo de trabajadores con discapacidad de la ciudad de Cali, a partir de cinco dimensiones: la estabilidad laboral, la jornada laboral, el ingreso laboral, la seguridad social y el ajuste al empleo.

\section{Metodología}

Se realizó un estudio cuantitativo, descriptivo y transversal. El registro parcial de datos sobre la participación de las personas con discapacidad en el mercado laboral conllevó a no tener un universo claramente definido; por lo tanto, se realizó un muestreo no probabilístico por cuotas, aplicado a 160 trabajadores con discapacidad de la ciudad de Cali. 
Las cuotas se definieron a partir del sexo y el tipo de discapacidad (física, visual, auditiva, mental o intelectual). La distribución del tipo de discapacidad se calculó teniendo en cuenta la prevalencia de la población con discapacidad para Cali, según datos del censo de 2005 (Departamento Administrativo Nacional de Estadística [DANE], 2005).

La encuesta de tipo transversal se diseñó a partir del registro de localización y caracterización para personas con discapacidad del DANE y la encuesta «Condiciones de trabajo de colombianos en Barcelona» (Roa, 2002). Se realizó prueba piloto y ajuste al instrumento. Este último comprendió seis módulos: el sociodemográfico, de antecedentes laborales, de estructura ocupacional, la descripción de la discapacidad, de condiciones de empleo y de condiciones de trabajo.

Se realizó un muestreo de bola de nieve exponencial no discriminativo, en el cual cada trabajador con discapacidad contactado proporcionó referidos y cada referido, a su vez, refirió a otros sujetos hasta conseguir la totalidad de los participantes. Los criterios de selección de la muestra fueron: manifestar interés por participar en la investigación, ser trabajador con discapacidad, ser mayor de edad y trabajar en la ciudad de Cali. Los datos se analizaron por medio del programa estadístico Statistical Package for the Social Sciences, SPSS, versión 22.

A partir de los datos recolectados, se construyó un índice de condiciones de empleo, el cual fue creado para medir las diferencias en la magnitud de un grupo de variables que se quieren relacionar efectivamente con las condiciones de empleo; en este sentido, es un instrumento de medida compuesto por una serie de variables que representan diferentes características y que proporcionan información sobre las personas que han sido parte del estudio. Es importante destacar que el índice es pertinente para el estudio de dimensiones complejas y heterogéneas de la vida social, puesto que son este tipo de dimensiones en la medida que requieren la combinación de variables de diferente naturaleza.

Con el propósito de lograr pertinencia y validez del índice, se siguieron los siguiente pasos: primero, delimitar el objetivo del índice a partir del cuerpo teórico utilizado en la investigación, en este sentido el índice representa toda la conceptualización a partir de la presencia de dimensiones de las condiciones de empleo; posteriormente, hubo una selección cuidadosa de las variables que conformarían las diferentes dimensiones escogidas, variables que fueron seleccionadas en función de la relevancia y de la capacidad que tenían para conformar esa dimensión. En este sentido, la selección de las variables está claramente soportada por decisiones teóricas.

El índice que se construyó transforma las variables en variables numéricas binarias, donde o representa la ausencia del atributo y 1 la presencia del mismo. Finalmente, este índice es una sumatoria de los diferentes atributos que se quieren 
medir, de forma tal que los sujetos se mueven en calificación de o a 1, donde o son las peores condiciones de empleo y 1 las mejores condiciones posibles en torno a las dimensiones escogidas (ver apéndice).

Para efectos de la descripción y análisis de las variables, se realizaron pruebas de normalidad K-S, y se encontró que las variables de las dimensiones no tienen un comportamiento normal. Esto significa que los estadísticos usuales, como la media y la desviación estándar, están distorsionados, por lo tanto, no se puede hacer uso de ellos para describirlas. Se emplearon como medidas más estables la mediana y medida de dispersión del rango intercuartílico.

Finalmente, la investigación fue sometida al Comité Institucional de Ética $\mathrm{Hu}-$ mana de la Universidad del Valle.

\section{Resultados}

Se presentarán los resultados a partir de cinco dimensiones: estabilidad laboral, jornada laboral, ingresos, ajuste del empleo y seguridad social. Cada una de ellas la conforma una serie de variables. A su vez, el análisis en cada dimensión se segmentó teniendo en cuenta el tipo de discapacidad, el tipo de ocupación, el sexo y la dependencia laboral de los trabajadores.

\section{Dimensión de estabilidad laboral}

La dimensión de estabilidad laboral hace referencia a las garantías que deben tener los trabajadores para permanecer en sus puestos de trabajo. Se construyó a partir de tres variables: tipo de contrato, antigüedad en el empleo y pluriempleo.

El box plot evidencia una asimetría negativa, notándose que la estabilidad laboral se concentra hacia el puntaje mayor. La distribución de la mediana de 0,83 con un rango intercuartílico de 0,5 indica la presencia de alta estabilidad laboral en los trabajadores con discapacidad. Solo alrededor de un $25 \%$ de trabajadores presenta bajos niveles de estabilidad laboral, la cual fluctúa entre o y o,5 (ver Gráfica 1).

Ahora bien, es importante el comportamiento de la dimensión según el tipo de discapacidad. Los trabajadores con discapacidad física son los que presentan mayor estabilidad laboral, con una mediana de o,83 y un rango intercuartílico de 0,5. Por su parte, los trabajadores con discapacidad mental y cognitiva comparten el mismo valor de mediana $(0,75)$, con una estabilidad laboral media; y los que menos estabilidad laboral tienen son los trabajadores con discapacidad auditiva y visual, con una mediana de o,67 (ver Tabla 2). 
GRÁFICA 1 | Box plot dimensión de estabilidad laboral

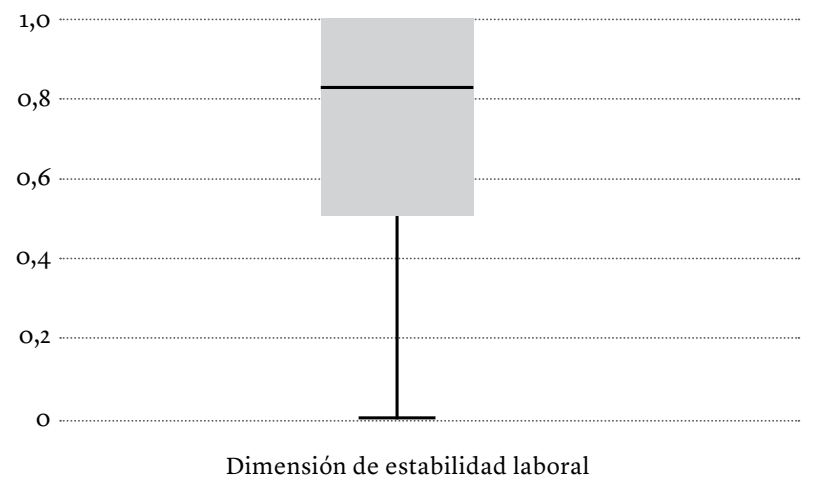

Fuente: elaboración propia.

Para densificar el análisis, se establece la comparación según el nivel de calificación de la ocupación y se encuentra que las ocupaciones no calificadas son las que presentan mayor estabilidad laboral (mediana 0,83 ). Por su parte, ocupaciones calificadas y semicalificadas comparten el mismo valor de mediana (o,67) (Ver tabla 2).

Estos datos pueden estar evidenciando que la alta estabilidad laboral en ocupaciones no calificadas está relacionada con la dependencia laboral y no necesariamente con el nivel de calificación de la ocupación. Los trabajadores calificados, generalmente, son trabajadores asalariados, mientras los trabajadores no calificados se insertan en mayor proporción al mercado por medio de la creación de sus negocios, crean sus propios nichos que les generan estabilidad, pero en la informalidad.

Se realizó la prueba U de Mann-Whitney para muestras independientes, con el objetivo de determinar la existencia de una relación estadísticamente significativa entre el nivel de calificación de la ocupación y la estabilidad laboral, porque los datos revelan de forma contraria que los trabajadores que desempeñan ocupaciones no calificadas presentan mayores niveles de estabilidad. La prueba $\mathrm{U}$ de $\mathrm{M}-\mathrm{W}$, efectivamente, muestra que sí hay asociación (ver Tabla 1).

En el mismo orden de ideas, los trabajadores independientes o empleadores son los que mayor estabilidad laboral tienen, con una mediana de 1 y un rango intercuartílico de o,5 en comparación con los asalariados. Los trabajadores independientes crean su propio nicho laboral, de manera que la estabilidad laboral es de otro orden, y no está mediada por el tipo de contrato. Su forma de medirla, según este índice, es por medio de la antigüedad en el empleo y la presencia de pluriempleo. 
TABLA 1 Prueba U de Mann-Whitney

Hipótesis nula

La distribución de dimensión de estabilidad laboral es

la misma entre las categorías de dependencia laboral

\begin{tabular}{ccc}
$\begin{array}{c}\text { Nivel de calificación } \\
\text { de la ocupación }\end{array}$ & Sig. & Decisión \\
\hline Ocupaciones calificadas & 0,896 & Conserve la hipótesis nula \\
\hline Ocupaciones semicalificadas & 0,780 & Conserve la hipótesis nula \\
\hline Ocupaciones no calificadas & 0,025 & Rechace la hipótesis nula
\end{tabular}

Fuente: elaboración propia,

Finalmente, se encuentran diferencias en la estabilidad laboral según el sexo, puesto que los hombres con discapacidad tienen empleos más estables, en comparación con las mujeres con discapacidad.

TABLA 2 Estabilidad laboral según tipo de discapacidad, sexo, nivel de calificación de la ocupación y dependencia laboral

\begin{tabular}{|c|c|c|c|}
\hline Sexo & \multicolumn{3}{|c|}{ Dimensión de estabilidad laboral } \\
\hline & $\mathbf{n}$ & Mediana & $\mathbf{R I}$ \\
\hline Hombre & 82 & 0,83 & 0,38 \\
\hline Mujer & 78 & 0,66 & 0,50 \\
\hline Total & 160 & 0,83 & 0,50 \\
\hline \multicolumn{4}{|l|}{ Tipo de discapacidad } \\
\hline Discapacidad física & 56 & 0,83 & 0,50 \\
\hline Discapacidad intelectual & 24 & 0,75 & 0,33 \\
\hline Discapacidad mental & 16 & 0,75 & 0,46 \\
\hline Discapacidad auditiva & 24 & 0,67 & 0,33 \\
\hline Discapacidad visual & 40 & 0,67 & 0,50 \\
\hline Total & 160 & 0,83 & 0,50 \\
\hline
\end{tabular}

Nivel de calificación de la ocupación

Ocupaciones calificadas

48

0,67

0,33 


\begin{tabular}{|c|c|c|c|}
\hline Ocupaciones semicalificadas & 36 & 0,67 & 0,50 \\
\hline Ocupaciones no calificadas & 76 & 0,83 & 0,33 \\
\hline Total & 160 & 0,83 & 0,50 \\
\hline \multicolumn{4}{|l|}{ Dependencia laboral } \\
\hline Asalariado & 104 & 0,83 & 0,33 \\
\hline Empleador o trabajador independiente & 56 & 1 & 0,50 \\
\hline Total & 160 & 0,83 & 0,50 \\
\hline
\end{tabular}

\section{Dimensión de jornada laboral}

La dimensión de jornada laboral hace referencia al tiempo de dedicación en la ejecución del trabajo. Se construyó a partir de tres variables: el número de horas trabajadas a la semana, la presencia o no de trabajo nocturno, y el trabajo por turnos.

El box plot evidencia una distribución simétrica de la dimensión. La distribución de la mediana es de 0,67 con un rango intercuartílico de 0,33 , que indica una presencia de alrededor de $50 \%$ de trabajadores con jornadas laborales adecuadas (48 horas aproximadamente), sin trabajo nocturno y por turnos. El bigote hacia abajo denota alrededor de un $25 \%$ de trabajadores con jornadas laborales poco adecuadas, jornadas intensas, incompletas, presencia de trabajo nocturno o por turnos, que fluctúan entre o y o,5. El bigote hacia arriba muestra la concentración de trabajadores con jornadas labores muy adecuadas que fluctúan entre 0,83 y 1 .

De acuerdo al tipo de discapacidad, al tipo de ocupación y a la dependencia laboral, no se encuentran diferencias en la mediana. Todos comparten el mismo valor de 0,67; sin embargo, se encuentran diferencias en los rangos intercuartílicos.

Según el tipo, los trabajadores con discapacidad auditiva presentan mayor dispersión en sus valores, con un rango intercuartílico de 0,50; es decir, presencia de trabajadores con adecuadas y poco adecuadas jornadas laborales. Mientras que los trabajadores con discapacidad visual tienen mayor uniformidad porque presentan el rango intercuartílico más pequeño $(0,29)$ (ver Tabla 3).

El comportamiento de la mediana y los rangos intercuartílicos para el nivel de calificación de la ocupación y el sexo no presenta diferencias significativas.

De acuerdo a la dependencia laboral, los trabajadores asalariados tienen mayor dispersión (rango intercuartílico de 0,50 ), de tal forma que se encuentran trabajadores con jornadas laborales adecuadas y otros con jornadas laborales poco adecuadas. 
GRÁFICA 2 | Boxplot dimensión de jornada laboral

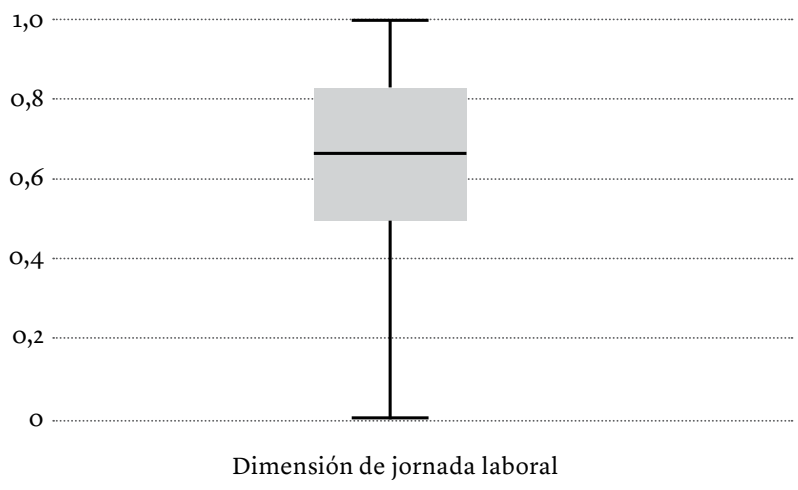

Fuente: elaboración propia.

En cambio, los trabajadores independientes presentan mayor uniformidad porque tienen un rango intercuartílico más pequeño $(0,17)$ (ver Tabla 3$)$. \begin{tabular}{l|l} 
TABLA 3 & $\begin{array}{l}\text { Jornada laboral según tipo de discapacidad, ocupación } \\
\text { y dependencia laboral }\end{array}$
\end{tabular}

\begin{tabular}{|c|c|c|c|}
\hline Sexo & \multicolumn{3}{|c|}{ Jornada laboral } \\
\hline & $\mathbf{n}$ & Mediana & RI \\
\hline Hombre & 82 & 0,67 & 0,33 \\
\hline Mujer & 78 & 0,67 & 0,21 \\
\hline Total & 160 & 0,67 & 0,33 \\
\hline \multicolumn{4}{|l|}{ Tipo de discapacidad } \\
\hline Discapacidad física & 56 & 0,67 & 0,42 \\
\hline Discapacidad intelectual & 24 & 0,67 & 0,33 \\
\hline Discapacidad mental & 16 & 0,67 & 0,38 \\
\hline Discapacidad auditiva & 24 & 0,67 & 0,50 \\
\hline Discapacidad visual & 40 & 0,67 & 0,29 \\
\hline Total & 160 & 0,67 & 0,33 \\
\hline
\end{tabular}




\begin{tabular}{|c|c|c|c|}
\hline \multicolumn{4}{|l|}{ Nivel de calificación de la ocupación } \\
\hline Ocupaciones calificadas & 48 & 0,67 & 0,29 \\
\hline Ocupaciones semicalificadas & 36 & 0,67 & 0,29 \\
\hline Ocupaciones no calificadas & 76 & 0,67 & 0,33 \\
\hline Total & 160 & 0,67 & 0,33 \\
\hline \multicolumn{4}{|l|}{ Dependencia laboral } \\
\hline Asalariado & 104 & 0,67 & 0,50 \\
\hline Empleador o trabajador independiente & 56 & 0,67 & 0,17 \\
\hline Total & 160 & 0,67 & 0,33 \\
\hline Total & 160 & 0,83 & 0,50 \\
\hline \multicolumn{4}{|l|}{ Nivel de calificación de la ocupación } \\
\hline Ocupaciones calificadas & 48 & 0,67 & 0,33 \\
\hline
\end{tabular}

\section{Dimensión ingreso laboral}

La dimensión de ingreso laboral hace referencia a la suma de dinero que se le otorga al trabajador por la labor realizada y la satisfacción que manifiesta por el ingreso. Se construyó a partir de dos variables: el ingreso mensual promedio por la labor realizada y el nivel de satisfacción frente al mismo.

Se evidencia una distribución asimétrica positiva, notándose mayor concentración hacia los valores mínimos. La mediana de o,5o con un rango intercuartílico de o,5o demuestra que alrededor del $75 \%$ de los datos se encuentran en una escala de 0,5 a 1 , lo que denota que los trabajadores se encuentran con unos ingresos laborales y una satisfacción hacia sus ingresos en un nivel medio y adecuado. El bigote hacia abajo demuestra que un $25 \%$ de los trabajadores puede estar con ingresos laborales bajos e insatisfechos con sus ingresos.

Los trabajadores con discapacidad física son los únicos con una mediana de 1 y un rango intercuartílico 0,50 que denotan los mejores ingresos y satisfacción del ingreso del grupo de trabajadores. El resto del grupo presenta una mediana de o,5o con diferencias en los rangos intercuartílicos. Según el nivel de calificación de la ocupación, se encuentra una clara relación: a mayor nivel de calificación de la ocupación, mayor es el ingreso y la satisfacción con él. Por su parte, los ingresos de las ocupaciones semicalificadas y no calificadas comparten el valor de la mediana $(0,50)$ y el rango intercuartílico $(0,50)$. 
GRÁFICA 3 | Boxplot dimensión de ingreso laboral

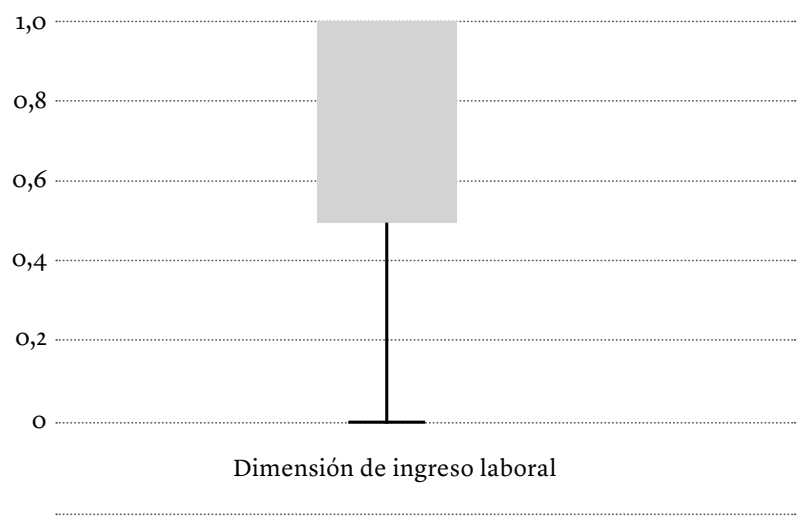

Fuente: elaboración propia.

De acuerdo a la dependencia laboral, son los trabajadores asalariados con discapacidad quienes presentan mayores ingresos y satisfacción con el mismo (mediana 0,75 ), en comparación con los independientes (mediana 0,50 ). Finalmente, no se encuentran diferencias salariales entre hombres y mujeres.

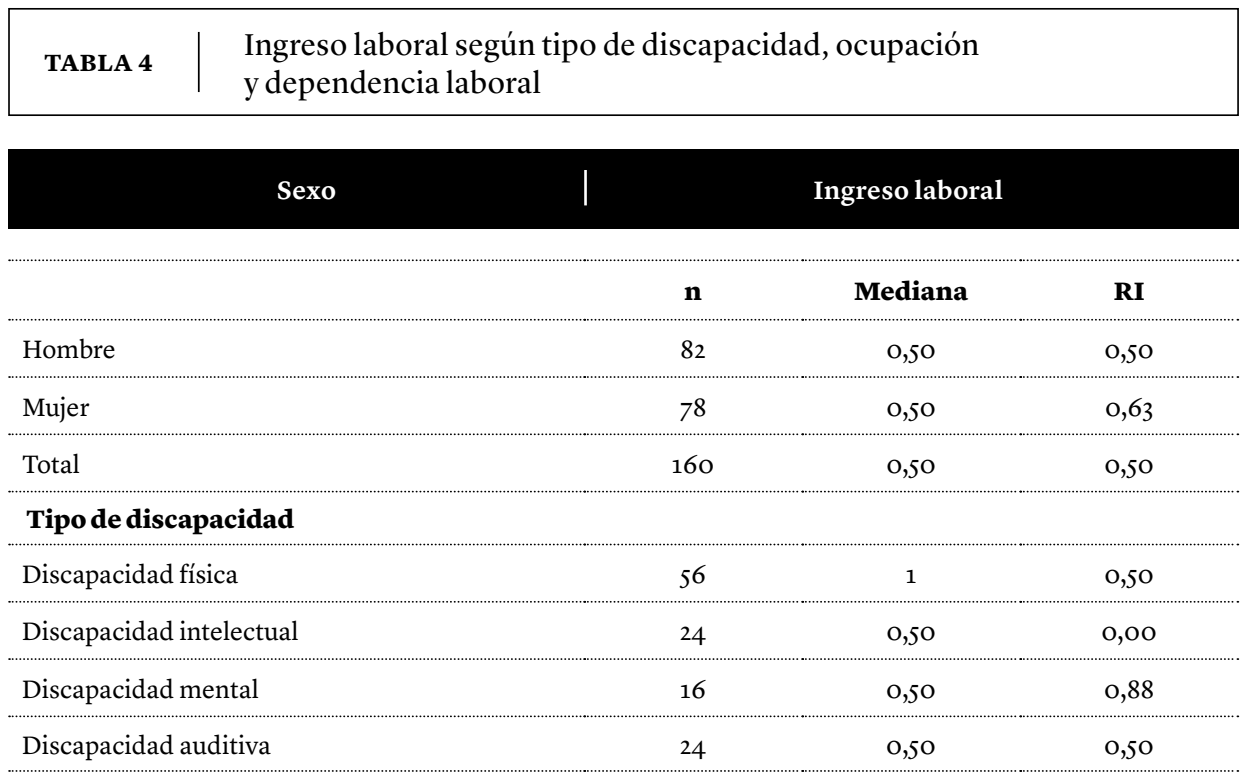




\begin{tabular}{|c|c|c|c|}
\hline Discapacidad visual & 40 & 0,50 & $1, \mathrm{OO}$ \\
\hline Total & 160 & 0,50 & 0,50 \\
\hline \multicolumn{4}{|l|}{ Nivel de calificación de la ocupaciones } \\
\hline Ocupaciones calificadas & 48 & $1, \mathrm{O}$ & 0,50 \\
\hline Ocupaciones semicalificadas & 36 & 0,50 & 0,50 \\
\hline Ocupaciones no calificadas & 76 & 0,50 & 0,50 \\
\hline Total & 160 & 0,50 & 0,50 \\
\hline \multicolumn{4}{|l|}{ Dependencia laboral } \\
\hline Asalariado & 104 & 0,75 & 0,50 \\
\hline Empleador o trabajador independiente & 56 & 0,50 & 0,50 \\
\hline Total & 160 & 0,50 & 0,50 \\
\hline
\end{tabular}

\section{Dimensión de seguridad social}

La dimensión de protección social hace referencia al acceso a la seguridad social integral por vía del trabajo. Se construyó a partir de tres variables: acceso a salud, pensiones y riesgos laborales.

En el box plot se evidencia una asimetría negativa, notándose que hay mayor concentración hacia el puntaje mayor. La distribución de la mediana de o,67 con un rango intercuartílico de 1 indica la presencia de alrededor de $50 \%$ de trabajadores con una seguridad social adecuada. Sin embargo, la no presencia de bigotes hacia abajo puede denotar la presencia de un grupo importante de trabajadores con bajo acceso a la seguridad social.

Al realizar el análisis por tipo de discapacidad, se encuentran diferencias entre el grupo de trabajadores. Aquellos con discapacidad auditiva son los que presentan mayor acceso a seguridad social, seguidos de los trabajadores con discapacidad intelectual. Los trabajadores con discapacidad mental son los que menos acceso tienen a la seguridad social.

De acuerdo al nivel de calificación de la ocupación, se nota claramente la relación directa entre el acceso o no a seguridad social y el nivel de calificación de la ocupación. A mayor calificación, mayor presencia de trabajos con acceso a seguridad social; a menor calificación de la ocupación, menor acceso a seguridad social. De alguna manera, esto puede estar indicando que las ocupaciones más calificadas están relacionadas con contratos que garantizan el acceso a la seguridad social o con ocupaciones que generen altos ingresos que permitan asegurarse (ver Tabla 5). 
GRÁFICA 4 | Boxplot dimensión de seguridad social

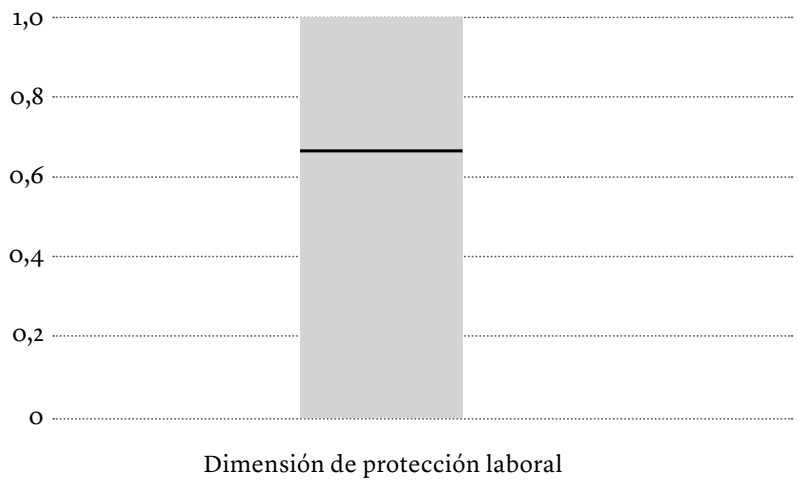

Fuente: elaboración propia.

Por otro lado, es clara la diferencia entre los trabajadores de acuerdo a su dependencia laboral. Los asalariados tienen trabajos que posibilitan el acceso a la seguridad social con una mediana de 1 ; mientras que los independientes tienen trabajos que no posibilitan el acceso a seguridad social, con una mediana de o. Con relación al sexo, no se encuentran diferencias significativas (ver Tabla 5).

\section{\begin{tabular}{l|l} 
TABLA 5 & Seguridad social según tipo de discapacidad, ocupación
\end{tabular} y dependencia laboral}

\begin{tabular}{|c|c|c|c|}
\hline Sexo & \multicolumn{3}{|c|}{ Protección laboral } \\
\hline & $\mathbf{n}$ & Mediana & RI \\
\hline Hombre & 82 & 0,67 & $1, \mathrm{oo}$ \\
\hline Mujer & 78 & 0,67 & $1, \mathrm{oo}$ \\
\hline Total & 160 & 0,67 & $1, \mathrm{oo}$ \\
\hline \multicolumn{4}{|l|}{ Tipo de discapacidad } \\
\hline Discapacidad física & 56 & 0,67 & $1, \mathrm{oo}$ \\
\hline Discapacidad intelectual & 24 & 0,83 & 1,00 \\
\hline
\end{tabular}




\begin{tabular}{|c|c|c|c|}
\hline Discapacidad mental & 16 & 0,17 & 0,67 \\
\hline Discapacidad auditiva & 24 & $1, \mathrm{OO}$ & 0,83 \\
\hline Discapacidad visual & 40 & 0,67 & 1,00 \\
\hline Total & 160 & 0,67 & $1, \mathrm{oo}$ \\
\hline \multicolumn{4}{|l|}{ Tipo de ocupaciones } \\
\hline Ocupaciones calificadas & 48 & $1, \mathrm{OO}$ & 0,33 \\
\hline Ocupaciones semicalificadas & 36 & 0,67 & $1, \mathrm{Oo}$ \\
\hline Ocupaciones no calificadas & 76 & o,oo & $1, \mathrm{oo}$ \\
\hline Total & 160 & 0,67 & $1, \mathrm{OO}$ \\
\hline \multicolumn{4}{|l|}{ Dependencia laboral } \\
\hline Asalariado & 104 & $1, \mathrm{OO}$ & 0,33 \\
\hline Empleador o trabajador independiente & 56 & 0,00 & 0,33 \\
\hline Total & 160 & 0,67 & $1, \mathrm{oo}$ \\
\hline
\end{tabular}

\section{Dimensión de ajuste al empleo}

La dimensión de ajuste al empleo se construyó a partir de dos variables: la relación entre la formación y el puesto de trabajo que ocupa el trabajador con discapacidad, y la posibilidad de ascenso.

En el box plot se evidencia una distribución simétrica de la dimensión. La distribución de la mediana de 0,75 con un rango intercuartílico de 0,50 indica una presencia de alrededor de $50 \%$ de trabajadores con empleos ajustados, es decir, con empleos acorde a la formación y con posibilidades de ascenso. El bigote hacia abajo denota alrededor de un $25 \%$ de trabajadores con empleos no ajustados, que fluctúan entre o y 0,5 .

De acuerdo al tipo, se encuentra que los trabajadores con discapacidad física y cognitiva son los que más ajuste al empleo tienen, con una mediana de 0,75 y un rango intercuartílico de 0,5 , mientras que los trabajadores con discapacidad mental son los que menos ajuste al empleo tienen, con una mediana de 0,13 y un rango intercuartílico de 0,75 .

Según el nivel de calificación de la ocupación, son los trabajadores con ocupaciones calificadas los que más presentan ajuste al empleo, con una mediana de 0,75 y un rango intercuartílico de 0,50 . 
GRÁFICA 5 | Boxplot dimensión de ajuste del empleo

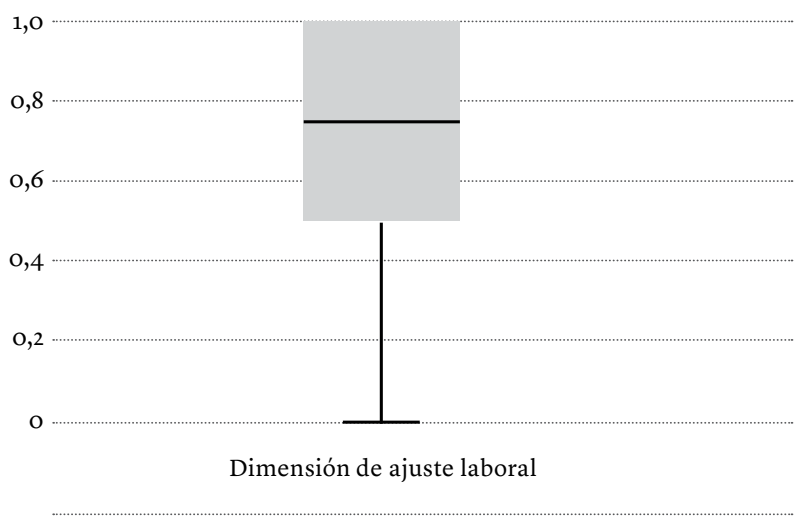

Fuente: elaboración propia.

Por otro lado, de acuerdo a la dependencia laboral, los trabajadores independientes o empleadores presentan mayor ajuste al empleo, lo cual llama la atención en la medida que la variable para los trabajadores independientes solo fue la relación entre el puesto de trabajo y la formación.

De acuerdo al sexo, se encuentran diferencias. Los hombres presentan mayor ajuste al empleo (mediana 0,75 ) en comparación con las mujeres (mediana 0,50 ).

\begin{tabular}{|l|l|}
\hline TABLA 6 & $\begin{array}{l}\text { Ajuste del empleo según tipo de discapacidad, ocupación, } \\
\text { dependencia laboral y sexo }\end{array}$ \\
\hline
\end{tabular}

\begin{tabular}{|c|c|c|c|}
\hline \multirow[t]{2}{*}{ Sexo } & \multicolumn{3}{|c|}{ Ajuste laboral } \\
\hline & $\mathbf{n}$ & Mediana & RI \\
\hline Hombre & 82 & 0,75 & 0,50 \\
\hline Mujer & 78 & 0,50 & 0,50 \\
\hline Total & 160 & 0,75 & $1, \mathrm{OO}$ \\
\hline \multicolumn{4}{|l|}{ Tipos de discapacidad } \\
\hline Discapacidad física & 56 & 0,75 & 0,50 \\
\hline Discapacidad intelectual & 24 & 0,75 & 0,50 \\
\hline
\end{tabular}




\begin{tabular}{|c|c|c|c|}
\hline Discapacidad mental & 16 & 0,13 & 0,75 \\
\hline Discapacidad auditiva & 24 & 0,63 & 0,50 \\
\hline Discapacidad visual & 40 & 0,63 & 0,69 \\
\hline Total & 160 & 0,75 & 0,50 \\
\hline \multicolumn{4}{|l|}{ Tipo de ocupaciones } \\
\hline Ocupaciones calificadas & 48 & 0,75 & 0,50 \\
\hline Ocupaciones semicalificadas & 36 & 0,50 & 0,44 \\
\hline Ocupaciones no calificadas & 76 & 0,50 & 0,50 \\
\hline Total & 160 & 0,75 & 0,50 \\
\hline \multicolumn{4}{|l|}{ Dependencia laboral } \\
\hline Asalariado & 104 & 0,50 & 0,25 \\
\hline Empleador o trabajador independiente & 56 & $1, \mathrm{OO}$ & $1, \mathrm{oo}$ \\
\hline Total & 160 & 0,75 & 0,50 \\
\hline
\end{tabular}

\section{Índice general de condiciones de empleo}

El índice de condiciones de empleo se calculó teniendo en cuenta las dimensiones anteriormente descritas. En este apartado se realizará el análisis en su conjunto.

En el box plot se evidencia la presencia de outliers, denotando un caso con muy bajas condiciones de empleo. La asimetría es negativa, notándose que las condiciones de empleo tienden hacia el puntaje mayor. La distribución de la mediana de o,66 con un rango intercuartílico de 0,26 indica una presencia de alrededor de $50 \%$ de trabajadores con buenas condiciones de empleo. El bigote hacia abajo denota alrededor de un $25 \%$ de trabajadores con malas condiciones de empleo, entre $0,2 \mathrm{y}$ 0,5 . El bigote hacia arriba muestra la concentración de trabajadores con muy buenas condiciones de empleo, que fluctúan entre o,79 y 1.

Los trabajadores con discapacidad física y auditiva son quienes presentan las mejores condiciones de empleo, con medianas de o,71 y o,70, respectivamente. Por su parte, los trabajadores con discapacidad mental son los que peores condiciones de empleo tienen, con una mediana de 0,56 y un rango intercuartílico de o,29.

De acuerdo al nivel de calificación de la ocupación, las condiciones de empleo guardan relación de mayor a menor, es decir, que entre más calificada la ocupación, mejores son las condiciones de empleo y entre menos calificada la ocupación, peores las condiciones de empleo (ver Tabla 7). 
GRÁFICA 6 | Boxplot índice de condiciones de empleo

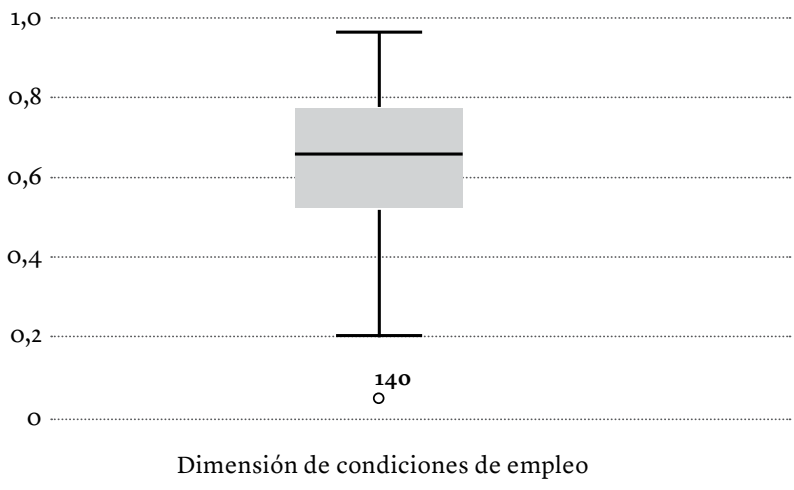

Fuente: elaboración propia.

Por otro lado, la dependencia laboral muestra que los trabajadores asalariados (con una mediana de 0,71 y un rango intercuartílico de 0,19 ) tienen mejores condiciones de empleo que los independientes (con una mediana de o,55 y un rango intercuartílico de o,26).

Finalmente, no se encuentra una diferencia estadísticamente significativa entre las condiciones de empleo de hombres y mujeres, sin embargo, las condiciones de los hombres son un poco mejores que las de las mujeres.

\begin{tabular}{|l|l|}
\hline TABLA 7 & $\begin{array}{l}\text { Índice de empleo según tipo de discapacidad, ocupación y } \\
\text { dependencia laboral }\end{array}$ \\
\hline
\end{tabular}

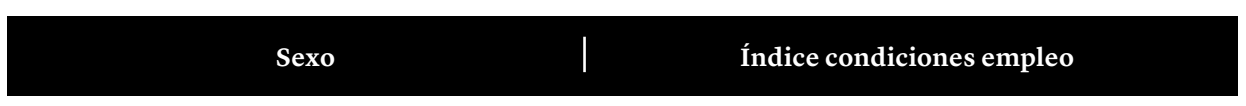

\begin{tabular}{lcccc} 
& & $\mathbf{n}$ & Mediana & RI \\
\hline Hombre & 82 & 0,67 & 0,30 \\
\hline Mujer & 78 & 0,65 & 0,25 \\
\hline Total & 160 & 0,66 & 0,26
\end{tabular}

Tipo de discapacidad

\begin{tabular}{|c|c|c|c|}
\hline Discapacidad física & 56 & 0,71 & 0,21 \\
\hline Discapacidad intelectual & 24 & 0,63 & 0,28 \\
\hline
\end{tabular}




\begin{tabular}{|c|c|c|c|}
\hline Discapacidad mental & 16 & 0,56 & 0,29 \\
\hline Discapacidad auditiva & 24 & 0,70 & 0,18 \\
\hline Discapacidad visual & 40 & 0,62 & 0,38 \\
\hline Total & 160 & 0,66 & 0,26 \\
\hline \multicolumn{4}{|l|}{ Tipo de ocupación } \\
\hline Ocupaciones calificadas & 48 & 0,76 & 0,19 \\
\hline Ocupaciones semicalificadas & 36 & 0,65 & 0,29 \\
\hline Ocupaciones no calificadas & 76 & 0,62 & 0,25 \\
\hline Total & 160 & 0,66 & 0,26 \\
\hline \multicolumn{4}{|l|}{ Dependencia laboral } \\
\hline Asalariado & 104 & 0,71 & 0,19 \\
\hline Empleador o trabajador independiente & 56 & 0,55 & 0,25 \\
\hline Total & 160 & 0,66 & 0,26 \\
\hline
\end{tabular}

\section{Conclusiones}

En términos de la estabilidad laboral, la mayoría de los trabajadores con discapacidad estudiados tienen empleos estables, han construido trayectorias laborales continuas y tienen vinculación laboral por medio de contratos. Sin embargo, cabe la posibilidad de cuestionar la complejidad de la dimensión de la estabilidad laboral, dado que se evidencia cierto tipo de vinculación laboral con una estabilidad que no está regida por el estatus del contrato. En mercados laborales regulares, el contrato es una forma de garantizar la estabilidad laboral de los trabajadores, pero en mercados laborales desregularizados, como el de Cali, esta estabilidad no está mediada del todo por elementos formales, como el contrato laboral. Además, esto puede estar indicando la adscripción a nichos laborales informales que proveen estabilidad sin la mediación de un contrato.

Los hombres con discapacidad tienen empleos más estables que las mujeres. Esta diferencia según el sexo puede estar denotando una doble vulneración.

El tipo de discapacidad incide en las características de la inserción laboral. Se encontró que, del conjunto de trabajadores participantes en la investigación, los trabajadores con discapacidad física presentan mayor estabilidad laboral. Por su parte, aquellos con discapacidad intelectual y mental presentan una estabilidad la- 
boral media, caracterizada por empleos por encima del año de antigüedad, pero con formas de contratación poco favorables. Los que presentan una menor estabilidad laboral son los trabajadores con discapacidad auditiva y visual.

Contrario a lo que podría esperarse, las ocupaciones no calificadas son las que presentan mayor estabilidad laboral. Según el test de U de Mann-Whitney, existe una asociación estadísticamente significativa entre estabilidad laboral y calificación de la ocupación.

Los trabajadores independientes o empleadores presentan mayor estabilidad laboral en comparación con los asalariados. Además, aquellos trabajadores crean sus propios nichos laborales. Finalmente, la estabilidad laboral no está mediada por el tipo de contrato y su forma de medirla, sino por medio de la antigüedad en el empleo y la no presencia de pluriempleo.

Con relación a la jornada laboral, prevalecen los trabajadores con discapacidad con jornadas labores adecuadas y muy adecuadas, es decir, con jornadas laborales no mayores a 48 horas, aproximadamente, sin trabajo nocturno o por turnos.

En términos generales, el comportamiento de la dimensión de la jornada laboral refleja aspectos que tienen que ver con la misma condición de salud de los trabajadores. Las largas jornadas laborales pueden deteriorar sus condiciones de salud. Por ejemplo, para un trabajador con discapacidad física, permanecer por mucho tiempo sentado puede originar lesiones cutáneas, y para un trabajador con discapacidad mental, puede generar carga emocional. Por otro lado, la poca presencia de trabajo nocturno y por turnos puede estar relacionada con el tipo de ocupación que realizan, pero también con la condición de salud y los imaginarios sociales que se construyen alrededor de la discapacidad. Permanecer en un entorno que constantemente señala la poca capacidad para desempeñar una actividad y los riesgos que de estas se deriva puede asociar el trabajo nocturno con un contexto poco protector para trabajadores con discapacidad, lo cual, quizás, pueda estar más asociado a trabajadores con discapacidad intelectual. Pagan (2017) encontró que existen variables, como obtener atención médica, descansar o el tiempo de consumo en el transporte, que inciden en que los trabadores con discapacidad no opten por jornadas laborales extensas.

En la dimensión del ingreso laboral, es importante enfatizar en la magnitud subjetiva que tiene el trabajo (Lindon, 2003). Al realizar un análisis univariado del ingreso mensual y la satisfacción con el mismo, se encontró que el 57,5\% recibe ingresos de hasta 1 SMMLV; el 23,8 \% entre 1 y 2 SMMLV; y el 18,8 \% más de 2 SMMLV, lo cual denota, al igual que en otras investigaciones, la presencia de bajos salarios (Rodríguez, Malo y Cueto, 2012). Sin embargo, al indagar por la satisfacción frente al ingreso, se encontró que el 66,9 \% se encuentra entre satisfecho y muy satisfecho con él. Esto pone en evidencia que, pese a la presencia de bajos ingresos mensuales, un número 
mayoritario de los trabajadores se encontraban muy satisfechos y satisfechos con el ingreso recibido. El índice arrojó la presencia de ingresos laborales adecuados y medios, dado que se tuvo en cuenta la dimensión subjetiva del trabajador.

Esta situación puede ser propia de los grupos vulnerables que han sido tradicionalmente excluidos del mercado de trabajo. El trabajo es una experiencia vital de inclusión social que va más allá de la subsistencia, razón por la cual el trabajador puede sentirse satisfecho con sus ingresos, así no sean adecuados, porque le permiten reforzar su autonomía y confianza. En este sentido, la satisfacción se fundamenta en una racionalidad instrumental (Bates, Goodley y Runswick-Cole, 2017; Pagan, 2011).

Se encontraron diferencias significativas según el tipo de discapacidad, el nivel de calificación de la ocupación y la dependencia laboral. Los trabajadores con discapacidad física tienen mejores ingresos y satisfacción del ingreso que el resto de la población con discapacidad. Los resultados encontrados revelaron que los trabajadores con discapacidad intelectual y auditiva son los que presentan menores ingresos. Este hallazgo guarda relación con la investigación de Díaz (2014).

Existe relación entre el nivel de calificación de la ocupación y el ingreso: a mayor nivel de calificación mayor es el ingreso y la satisfacción con el mismo. Por lo tanto, las ocupaciones calificadas presentan mejores ingresos y satisfacción. Los trabajadores asalariados con discapacidad presentan mayores ingresos y satisfacción con relación a los independientes.

Los trabajadores con discapacidad presentan heterogeneidad en el acceso a la seguridad social, ya que fluctúan entre tener garantizada la afiliación y no tenerla. La afiliación en salud es del 63,1 \% como cotizante, mientras que el $57 \%$ cotiza a pensiones, y se tiene una baja afiliación a riesgos laborales. Con el índice se encontraron diferencias significativas según el tipo de discapacidad, el nivel de calificación de la ocupación y la dependencia laboral, de manera que los trabajadores con discapacidad auditiva y cognitiva son los que presentan mayor protección social. Este grupo está ligado a los programas de inclusión laboral que ofrecen las empresas de supermercados, de tal manera que garantizan contratos con prestaciones sociales. Los trabajadores con discapacidad mental son los más desprotegidos, con una mediana cercana a 0 .

Existe una relación directa entre la presencia o no de acceso a seguridad social y el nivel de calificación de la ocupación. A mayor calificación de la ocupación mayor presencia de trabajos con acceso a seguridad social. Por su parte, los trabajadores asalariados tienen empleo con mayor seguridad social, esto puede indicar que el estatus del contrato que tiene el trabajador asalariado garantiza el acceso a la seguridad social. 
Con relación al ajuste al empleo, los trabajadores con discapacidad tienen, en su mayoría, empleos acordes con su formación y, a su vez, presentan como tendencia central pocas posibilidades de ascenso. Se encontraron diferencias según el tipo de discapacidad, el nivel de calificación, la dependencia laboral y el sexo.

El índice general de condiciones de empleo reveló que uno de cada dos trabajadores presenta buenas condiciones de empleo, y se encontraron diferencias significativas de acuerdo al tipo de discapacidad y a la dependencia laboral.

El tipo de discapacidad influye en la inserción laboral, pues la heterogeneidad del grupo revela que los trabajadores con discapacidad física son los que presentan mejores condiciones de empleo, en la medida en que tienen una mayor estabilidad en el empleo, reciben la mejor remuneración y presentan los empleos más ajustados. Por su parte, los trabajadores con discapacidad mental son los que tienen las peores condiciones de empleo, ya que presentan una estabilidad laboral media, se encuentran desprotegidos en el trabajo y con poco ajuste al empleo. Este hallazgo guarda relación con otras investigaciones que han encontrado que el tipo de discapacidad incide en la participación en el mercado de trabajo (Albarrán-Lozano y Alonso-González, 2010; Dávila, 2004; Díaz, 2014; Hernández y Millán, 2015; Naraharisetti y Castro, 2016).

La dependencia laboral influye en las condiciones de empleo de los trabajadores con discapacidad, de manera que los asalariados presentan mejores condiciones de empleo que los independientes, lo cual se refleja en los buenos ingresos y en tener empleos con acceso a seguridad social. Esto puede estar indicando que los trabajadores se independizan no por una decisión propia ni como parte de un proceso de ascenso laboral, sino porque no pueden acceder al empleo asalariado. Los trabajadores independientes pueden representar un síntoma de las rigideces del mercado de trabajo, donde la idea de independencia se vuelve sinónimo de autonomía y agencia, pero termina encubriendo la precariedad del trabajo informal y revelando que la independencia no es una elección, sino el único camino para la inserción laboral, debido a la discriminación por su discapacidad que percibe el grupo de trabajadores.

Finalmente, aunque no es estadísticamente significativa, se encuentra una leve diferencia de las condiciones de empleo entre hombres y mujeres, siendo los hombres quienes presentan mejores condiciones. Este hallazgo guarda relación con otras investigaciones que plantean que el género femenino se convierte en un factor de mayor discriminación y exclusión laboral en la población con discapacidad (Dávila, 2006; Díaz, 2014; Lobato, Shum, Portillo y Conde, 2006; Maldonado, 2011; Muñoz-Cantero, Novo-Corti y Rebollo-Quintela, 2013). 


\section{Anexo}

\section{Metodología del índice de condiciones de empleo}

La construcción del índice se realizó a partir de las cinco dimensiones establecidas para configurar las condiciones de empleo en esta investigación. A cada dimensión se le otorgó un porcentaje de acuerdo al nivel de importancia. En cada dimensión, se les asignó a las variables un valor entre o y 1.

Dimensión de estabilidad laboral: esta dimensión se construyó a partir de tres variables: la antigüedad en el empleo, la modalidad del contrato y la presencia de pluriempleo. Se le asignó el mayor porcentaje del conjunto de las dimensiones (30\%).

\begin{tabular}{|c|c|c|}
\hline Variable & Opciones de respuesta & $\begin{array}{l}\text { Valor asignado } \\
\text { para el índice }\end{array}$ \\
\hline \multirow{2}{*}{ Antigüedad en el empleo } & Más de un año en el empleo & 1 \\
\hline & Menos de un año en el empleo & $\mathrm{o}$ \\
\hline \multirow{5}{*}{ Modalidad del contrato } & Contrato laboral escrito a término indefinido & 1 \\
\hline & Contrato laboral escrito a término fijo & 0,5 \\
\hline & Contrato por prestación de servicios & 0,5 \\
\hline & Contrato verbal & $\mathrm{o}$ \\
\hline & No tiene contrato & $\mathrm{O}$ \\
\hline \multirow{2}{*}{ Pluriempleo } & Tiene otro empleo & $\mathrm{o}$ \\
\hline & No tiene otro empleo & 1 \\
\hline
\end{tabular}

Dimensión jornada laboral: esta dimensión se construyó a partir de tres variables: el trabajo por turnos, el trabajo nocturno y el promedio de horas trabajadas a la semana. Se le asignó el $15 \%$ del conjunto de las dimensiones.

\begin{tabular}{|c|c|c|}
\hline Variable & Opciones de respuesta & $\begin{array}{l}\text { Valor asignado } \\
\text { para el índice }\end{array}$ \\
\hline \multirow{2}{*}{ Trabajo por turnos } & Sí trabaja por turnos & $\mathrm{o}$ \\
\hline & No trabaja por turnos & 1 \\
\hline \multirow{3}{*}{ Trabajo nocturno } & Siempre trabaja por la noche & $\mathrm{o}$ \\
\hline & A veces trabaja por la noche & 0,5 \\
\hline & Nunca trabaja por la noche & 1 \\
\hline
\end{tabular}


semana

Entre 1 y 39 horas

Entre 40 y 50 hora

Más de 51 horas

No tiene otro empleo $\mathrm{o}$

1

O

1

Dimensión ingreso: esta dimensión se construyó a partir de dos variables: el ingreso mensual y la satisfacción frente al ingreso. Se le asignó el 20\% del conjunto de las dimensiones.

\begin{tabular}{|c|c|c|}
\hline Variable & Opciones de respuesta & $\begin{array}{l}\text { Valor asignado } \\
\text { para el índice }\end{array}$ \\
\hline \multirow{2}{*}{ Ingreso mensual } & Más de un salario mínimo & 1 \\
\hline & Menos de un salario mínimo & $\mathrm{O}$ \\
\hline \multirow{4}{*}{$\begin{array}{l}\text { Satisfacción con relación al } \\
\text { ingreso }\end{array}$} & Muy satisfecho & 1 \\
\hline & Satisfecho & 1 \\
\hline & Insatisfecho & $\mathrm{O}$ \\
\hline & Muy insatisfecho & $\mathrm{O}$ \\
\hline
\end{tabular}

Dimensión protección social: esta dimensión se construyó a partir de tres variables: la afiliación al sistema de salud, afiliación a pensiones y afiliación a la aseguradora de riesgos laborales. Se le asignó el 20\% del conjunto de las dimensiones.

\begin{tabular}{|c|c|c|}
\hline Variable & Opciones de respuesta & $\begin{array}{c}\text { Valor asignado } \\
\text { para el índice }\end{array}$ \\
\hline \multirow{4}{*}{ Afiliación al sistema de salud } & Cotizante & 1 \\
\hline & Régimen subsidiado & $\mathrm{o}$ \\
\hline & Beneficiario & o \\
\hline & No está afiliado & o \\
\hline \multirow{3}{*}{ Afiliación a pensiones } & A través de la empresa & 1 \\
\hline & No cotiza & o \\
\hline & A través del trabajador & 1 \\
\hline \multirow{2}{*}{ Afiliación a la ARL } & Si está afiliado & 1 \\
\hline & No está afiliado & o \\
\hline
\end{tabular}


Dimensión ajuste al empleo: esta dimensión se construyó a partir de dos variables: las posibilidades de ascenso del trabajador en su lugar de trabajo y la relación entre la formación y el trabajo que realiza. Se le asignó el $15 \%$ del conjunto de las dimensiones.

\begin{tabular}{|c|c|c|}
\hline Variable & Opciones de respuesta & $\begin{array}{l}\text { Valor asignado } \\
\text { para el índice }\end{array}$ \\
\hline \multirow{4}{*}{ Posibilidades de ascenso } & Muchas posibilidades & 1 \\
\hline & Alguna posibilidad & 0,5 \\
\hline & Poca posibilidad & o \\
\hline & Ninguna posibilidad & $\mathrm{o}$ \\
\hline \multirow{5}{*}{$\begin{array}{l}\text { Relación entre la formación } \\
\text { y el trabajo que realiza }\end{array}$} & Es el correcto & 1 \\
\hline & Es más bajo que mi formación & o \\
\hline & Realmente sería necesaria más formación & 1 \\
\hline & Necesitaría una formación distinta & 0,5 \\
\hline & No tiene otro empleo & 1 \\
\hline
\end{tabular}

\section{Referencias}

ALBARRÁN-LOZANO, I. y ALONSO-GONZÁLEZ, P. (2010). Participación en el mercado laboral español de las personas con discapacidad y en situación de dependencia. Papeles de población, $16(64), 218-256$.

ANATOL'EVNA, S. A. y NATigovnA, F. L. (2017). Pressing Issues of Disability Employment. Economic and Social Changes: Facts, 6(48), 126-142.

BATES, K., GOODLEY, D. y RUNSWICK-COLE, K. (2017). Precarious lives and resistant possibilities: the labor of people with learning disabilities in times of austerity. Disability \& Society, $32(2), 160-175$.

BOIAROV, S. (coord.). (2009). Telecapacitados: Teletrabajo para la inclusión laboral de las personas con discapacidad. Buenos Aires: El Cid Editor.

CANALS, G. (2003). La inserción de personas con discapacidad en la empresa ordinaria. Documentación social, 130, 229-248.

DÁVILA, C. D. (2006). Discapacidad y género. Un estudio de participación en el mercado de trabajo español. Moneda y Crédito, 223, 127-158. 
DÁVILA, D. (2004). El efecto de la discapacidad sobre la participación en el mercado de trabajo. Informe de investigación. Manuscrito inédito.

DEPARTAMENTO ADMINISTRATIVO NACIONAL DE ESTADÍSTICA. (2005). Censo general 2005. Recuperado de http://www.dane.gov.co/censo/files/libroCenso2oo5nacional.pdf

DÍAZ, E. (2014). Nuevas dinámicas de desigualdad y exclusión sociolaboral de las personas con discapacidad durante la crisis económica en España. Revista Chilena de Terapia Ocupacional, $14(2), 27-44$.

HERNÁNDEZ, J. y MILLÁN, J. M. (2015). Las personas con discapacidad en España: inserción laboral y crisis económica. Revista Española de Discapacidad (REDIS), 3(1), 29-56.

JANG, Y., WANG, Y. T. y LIN, M. H. (2014). Factors affecting employment outcomes for people with disabilities who received disability employment services in Taiwan. Journal of occupational rehabilitation, 24(1), 11-21.

LINDON, A. (2003). La precariedad laboral como experiencia a través de la narrativa de vida. Gaceta Laboral, 9(3), 333-352.

LOBATO, H., SHUM, G., PORTILLO, I. y CONDE, Á. (2006). Mujer, discapacidady empleo: tejiendo la discriminación. Huelva: Universidad de Huelva.

LÓPEZ, C. y SECO, E. (2005). Discapacidad y empleo en España: su visibilidad. INNOVAR. Revista de Ciencias Administrativas y Sociales, 15(26), 59-72.

MALDONADO, E. (2011). Eje «empleo sin barreras»: discursos y prácticas en la cotidianidad laboral. En X. Andrade (coord.), Discapacidades en Ecuador: Perspectivas criticas, miradas etnográficas (pp. 81-105). Quito: FLACSO.

MALDONADO, S. (2005). ¿Exclusión o discriminación? El caso de las personas con discapacidad en el mercado laboral peruano. Ophelimos, 47, 2-28.

MUÑOZ-CANTERO, J. M., NOVO-CORTI, I. y REBOLLO-QUINTELA, N. (2013). Análisis de las actitudes, de los jóvenes trabajadores del sector textil hacia la discapacidad: diferencias por razón de género. Revista de Investigación Educativa, 31(1), 93-115.

NACIONES UNIDAS (2007). Convención sobre los Derechos de las Personas con Discapacidady Protocolo Facultativo. Recuperado de http://www.un.org/spanish/disabilities/default.asp?id=497

NARAHARISETTI, R. y CASTRO, M. C. (2016). Factors associated with persons with disability employment in India: a cross-sectional study. BMC public health, 16(1), 1063.

PAGAN, R. (2011). Ageing and disability: Job satisfaction differentials across Europe. Social Science \& Medicine, 72(2), 206-215.

. (2017). Impact of Working Time Mismatch on Job Satisfaction: Evidence for German Workers with Disabilities. Journal of Happiness Studies, 18(1), 125-149. 
ROA, M. G. (2002). Mercado de trabajo y condiciones laborales de inmigrantes: colombianos en tres comarcas de Barcelona (Tesis doctoral). Universidad de Barcelona, Barcelona, España.

RODRÍGUEZ, C. y RICO, L. (2009). Discapacidad y derecho al trabajo. Bogotá: Universidad de Los Andes.

RODRÍGUEZ, F. (2010). Estudio diagnóstico sobre las barreras para la inclusión laboral. Recuperado de http://www.pactodeproductividad.com/pdf/documentodiagnosticopactodeproductividad.pdf

RODRÍGUEZ, V., MALO, M. A. y CUETO, B. (2012). Diferencias salariales por discapacidad y Centros Especiales de Empleo. Cuadernos de economía, 35(98), 100-116.

SOLSONA, D. A. (2018). Imaginarios sociales sobre la discapacidad en la región de Magallanes, Chile: de sutiles semánticas a prácticas instituidas. Intersticios. Revista sociológica de pensamiento crítico, 12(2), 95-108.

VORNHOLT, K., VILlOTTI, P., MUSCHALlA, B., BAUER, J., COLELlA, A., ZIJLSTRA, F. y CORBIÈRE, M. (2018). Disability and employment-overview and highlights. European journal of work and organizational psychology, 27(1), 40-55. 